\title{
A systematic scoping review of early interventions for parents of deaf infants
}

\author{
B. Wright ${ }^{1}$, R. Hargate ${ }^{1}$, M. Garside ${ }^{\text {1* }}$, G. Carr ${ }^{2}$, T. Wakefield ${ }^{3}$, R. Swanwick ${ }^{4}$, I. Noon ${ }^{3}$ and P. Simpson ${ }^{5}$
}

\begin{abstract}
Background: Over $90 \%$ of the 50,000 deaf children in the UK have hearing parents, many of whom were not expecting a deaf child and may require specialist support. Deaf children can experience poorer long-term outcomes than hearing children across a range of domains. After early detection by the Universal Newborn Hearing Screening Programme, parents in the UK receive support from Qualified Teachers of the Deaf and audiologists but resources are tight and intervention support can vary by locality. There are challenges faced due to a lack of clarity around what specific parenting support interventions are most helpful.

Methods: The aim of this research was to complete a systematic scoping review of the evidence to identify early support interventions for parents of deaf infants. From 5577 identified records, 54 met inclusion criteria. Two reviewers screened papers through three rounds before completing data extraction and quality assessment.

Results: Identified parent support interventions included both group and individual sessions in various settings (including online). They were led by a range of professionals and targeted various outcomes. Internationally there were only five randomised controlled trials. Other designs included non-randomised comparison groups, pre / post and other designs e.g. longitudinal, qualitative and case studies. Quality assessment showed few high quality studies with most having some concerns over risk of bias.

Conclusion: Interventions commonly focused on infant language and communication followed by parental knowledge and skills; parent wellbeing and empowerment; and parent/child relationship. There were no interventions that focused specifically on parent support to understand or nurture child socio-emotional development despite this being a well-established area of poor outcome for deaf children. There were few UK studies and research generally was not of high quality. Many studies were not recent and so not in the context of recent healthcare advances. Further research in this area is urgently needed to help develop evidence based early interventions.
\end{abstract}

Keywords: Deaf, Early intervention, Newborn Hearing Screening, Parents, Parent support intervention

\section{Background}

\section{Deaf children and current outcomes}

The World Health Organisation (WHO) reports that around 34 million children worldwide are deaf $(>40$ decibels hearing loss (dBHL) in the better hearing ear)

\footnotetext{
*Correspondence: megan.garside@nhs.net

${ }^{1}$ Leeds and York Partnership NHS Foundation Trust, COMIC Research,

IT Centre, Science Park, University of York, Innovation Way, Heslington, York YO10 5NP, UK

Full list of author information is available at the end of the article
}

[1]. The Consortium for Research into Deaf Education (CRIDE) survey estimated that there are at least 53,954 deaf (all levels of deafness, from mild to profound) children from birth up to the age of 19 years 11 months in the UK [2]. Over $90 \%$ of deaf children in the UK are born to hearing parents, most of whom were not expecting a deaf child $[3,4]$ and whom may require specialist support and advice.

There is a body of evidence that a significant number of deaf children experience poorer outcomes than hearing children, in terms of educational attainment [5], social 
domains [6] as well as poorer long term outcomes including increased unemployment [7]. Being deaf can have a range of impacts for both the child and their family [8] including auditory, linguistic, cognitive, social, literacy, and academic functioning [9]. Early language deprivation and language delay can lead to challenges in many developmental domains [10-16] including communication challenges $[17,18]$, poorer written language [19] and poor academic outcomes [20]. Additionally some deaf children can display difficulties in executive functioning [21], abstract thinking and problem-solving skills that adversely affect academic achievement [22].

\section{Social emotional development}

Language delay [23-25] thought to be associated with language deprivation $[26,27]$ can in turn lead to delays in Theory of Mind (ToM) (empathy-related) skills [14]. For example, research has shown deaf toddlers exchanging fewer social-communicative signals and having more difficulties understanding the intentions of others [28]. Social and emotional development provides the foundation for how people feel about themselves and experience interactions with others and begins at birth, continuing throughout the lifespan [29]. This unique pattern of social and emotional development may predispose to both serious social challenges and increased psychological distress $[15,30]$ and are related to a range of other poor socioemotional outcomes [17, 20,31].

Problems initiating and sustaining peer relationships and development of self-esteem [32] compound challenges in social settings. Social isolation and low popularity status during childhood have been shown to predict poor emotional well-being in the short term and future adverse consequences for mental health [14, 33]. Deaf children across England not in contact with mental health services have been found to have 2 to 3 times the rates of mental health problems compared to other children [34] and a systematic review found higher rates of behaviour problems in deaf children across numerous countries [35].

\section{Current international guidelines}

The Universal Newborn Hearing Screening Programme (UNHSP) was introduced across the UK between 2002 and 2006 [36] to screen all infants shortly after birth for signs that they may be deaf. Results to date suggest positive associated language [37] and health economic [38] outcomes. Early detection followed by specialist support programmes have been found to improve many negative outcomes for deaf children [39-41] including language acquisition and learning skills [29, 42, 43]. However, the outcomes for deaf children are highly variable due to many different factors including those described above [44].

On learning that their child is deaf, parents require a level of emotional support to adjust to this new information during the transition from diagnosis to early management $[45,46]$. Families must navigate through a myriad of services and choices including the NHS, local authorities and private agencies, and their own family constructs, all of which requires sensitivity to the social and emotional needs of a family with a deaf baby [47]. A consensus statement on early intervention lays out aspirations for good practice [48]. Current guidance is that early intervention for deaf infants should include parents as the most important agents for supporting their young children's language development [16] and that professionals who work with parents should focus on promoting their abilities to provide a language-rich environment [42, 48, 49]. Family-centred care has been advocated as the optimal way of addressing family needs in early intervention [50].

Most countries provide a range of support to families during early hearing detection and intervention programs. In the US parents receive information from an audiologist, written information and discussion with a medical professional [50]. Various states offer different additional support. For example in Colorado, USA all families of diagnosed children are referred to an early intervention system that begins with a counselling and information session with a deaf early intervention provider. Subsequently an early intervention co-ordinator consults with the family, establishes links to the local education pathways, offers access to sign language training for the family and engages them in a 6 month state wide program of support. This is offered to all families. However there has been no randomised controlled trial (RCT) to evaluate this to date, and no other published studies which met our PICOS criteria and so it does not appear in this review. In the UK Qualified Teachers of the Deaf (QToD) play an important role in providing early education services to families of infants and toddlers with hearing loss [51]. There is an element of universal provision but these services are often needs led depending on factors including audiological evaluation, child factors such as other significant medical conditions, social factors and parental choice. Current protocol in England is that every child identified by the UNHSP is contacted by a QToD within two working days post-referral [52]. The QToD works closely with the parent after the initial meeting and diagnostic period. This support is ongoing during the pre-school period. Formal parent support interventions are not often used but the regular QToD support is holistic, not just concentrating on language and communication but also on social and emotional 
development, audiological support and signposting to additional services if necessary. Many services will use the National Sensory Impairment Partnership (NatSIP) Eligibility Framework [53] and their specific early years' guidelines $[54,55]$ to determine levels of support according to individual need. This framework includes weighting of specific criteria but it is clear there still needs to be a focus on individual needs. However, provision is not universal, leading to variable offers for parents in different localities which may lead to deaf babies and young children of similar need getting inconsistent support due to a lack of resources despite the best efforts of specialist services. One study in Australia has reported such a challenge with many gaps in family-centred service provision at the time of identification and after enrolment in early hearing intervention services [18].

Many UK services have an Early Years (EYs) specialist QToD who works with these families at this stage. They may follow the Early Support Monitoring Protocol (revised from September 2020 as Success from the Start) but this is not mandatory, nor is it a formal intervention programme but a developmental resource used mainly to work with families to support them to observe, monitor and record progress their children make. QToDs receive some early years guidance during their training, delivered within the full training protocol.

\section{Barriers of access to support and eligibility criteria}

Even where intervention is available, families are extremely diverse and several factors may hinder families from accessing early intervention. These include language barriers with parents, lack of availability as well as cultural challenges during audiological testing (e.g., families sometimes preferred male practitioners, cultures where disability is stigmatised sometimes leading to caregivers denying the hearing loss or declining support or amplification for their children) [56].

Based on an ever-growing body of research, familycentred practices are a recommended, evidence-based principle of early childhood intervention but there continues to be a gap between recommendations and implementation of such practice across all disciplines [57].

\section{What are the gaps in the literature that we need to fill?}

A comprehensive synthesis of the available research evidence is necessary to inform a discussion about appropriate provision of support and interventions for parents of deaf infants. A well-established key criterion for the implementation of screening in society is the existence of a helpful and accepted intervention [58] which in the context of new-born hearing screening is early intervention with parents $[48,59]$.

\section{Methods}

The main aim of this review was to identify the available literature for early parenting interventions for deaf infants.

This research seeks to add to the evidence base by providing an up to date systematic scoping review of parent support interventions for deaf infants, synthesising the targets of these interventions and highlighting any evidence gaps to inform subsequent early intervention training and research in a UK context.

The review was registered on the PROSPERO database: CRD42019138001.

\section{Stakeholder workshops}

Prior to running literature searches the study team held national workshops with service providers and academics to gather information about the support that services currently offer parents of deaf infants aged 0-5 years old. The workshops included presentations and group discussions about good practice, the eligibility criteria for access to interventions, barriers to practice and gaps in research. The research team also held a meeting with parents of deaf infants to gather information about their experiences of the support they have been offered. Notes from discussion at these workshops and meetings were collated and key intervention elements and outcomes were identified, and included in the comprehensive search strategy for the systematic scoping review.

\section{Systematic scoping review}

Key stakeholders were identified from the workshops and invited to form an expert panel. The search strategy and PICOS criteria were developed with input from the workshops and finalised with the expert panel.

The final PICOS were as follows:

\section{Population}

- Deaf and/or hearing parents with children identified as deaf aged between birth and 5 years

- Children who are mild to profoundly deaf (between $40 \mathrm{~dB}$ to $140 \mathrm{~dB}$ on the audiogram in both ears) regardless of whether they are sign language users, or use hearing aids, have received cochlear implantation or use other communication aids

\section{Intervention}

- Any parenting intervention and/or support for this population 
For example; support for parents, parenting groups, skills training, provision of information, therapy groups, family therapy or language and communication support in a family context or delivered through the parent

- Any setting (e.g. home based, school, health centre, hospital) including both group and 1:1 interventions, with or without the involvement of the child

\section{Comparison}

- Where the study is a randomised controlled trial we included any comparator (control conditions or other active comparators)

- Other designs were included and we applied the same comparator criteria for any study using a comparison group (e.g. non-randomised controlled designs)

- Other empirical designs such as pre-post designs were included in the absence of a comparator

\section{Outcomes}

- Child and parent outcomes such as language acquisition and communicative development, socio-emotional outcomes (including mental health outcomes) and cognitive and educational outcomes

- Behavioural, quality of life outcomes or developmental milestones, as well as parenting outcomes such as studies measuring parental emotions, language, communication, stress and mental wellbeing

- Validated instruments were preferred where possible; however, studies measuring descriptive statistics were included where relevant (e.g. vocabulary obtained or educational outcomes)

\section{Study types}

- We considered all study designs (e.g. randomised and non-randomised intervention studies and observational studies including cohort, qualitative evaluations, descriptive, case-control, cross-sectional)

- Studies could be conducted in any country and no exclusions were made on the basis of publication status, date or language, we explored processes for translation where necessary. If conference abstracts were identified, they were included if they provided enough information for data extraction. If they did not, details were sought from the author or an available results paper.

\section{Search strategy}

A search strategy was designed to capture the population of parents/caregivers of children diagnosed as deaf and a range of interventions (based on existing knowledge and feedback from the stakeholder workshops). A full search strategy can be found in Additional file 1.

Searches were loaded into EndNote bibliographic software and then transferred to an Excel database for sifting. The searches were run by an information specialist at the Centre for Reviews and Dissemination (CRD, University of York) on the following databases in September 2019: CINAHL, Cochrane Central Register of Controlled Trials (CENTRAL), Embase, MEDLINE, PsycINFO, Science Citation Index, Scopus and Social Science Citation Index.

\section{Screening and data extraction}

Two independent reviewers conducted screening over three sifting stages. References of reviews, books, and included literature were checked to ensure all relevant papers were identified. Agreement for each sift was calculated over a minimum $10 \%$ overlap, and was consistently above the pre-specified $80 \%$. Disagreements were discussed and resolved with a third independent reviewer. Authors were contacted where necessary to clarify outcome measures and results.

For each eligible study, data was extracted by two reviewers using the Template for Intervention Description and Replication (TIDieR) checklist [60] as a guideline for descriptions of included interventions. The two reviewers conducted quality assessment using the Revised Cochrane Collaborations Assessment Tool for Assessing Risk of Bias in Randomised Trials (ROB-2) [61] tool for RCTs and the Risk Of Bias In Non-Randomised Studies of Interventions tool (ROBINS-I) [62] for non-randomised studies (comparison groups and pre-post study designs). Additional file 2 includes a table (Table 1) of characteristics for included studies in the review. This presents further detail on the data extracted.

\section{Synthesis}

For randomised controlled trial studies only, we compared these to the elements outlined in the International Consensus Statement [48] to determine how many of these 10 principles of best practise were included.

The final included studies were discussed at an expert panel meeting. At this meeting we discussed the most appropriate way to present the studies as well as the key outcomes to share. As a result, themes were identified, based on the primary focus of the intervention, and these have been used to summarise the studies. 


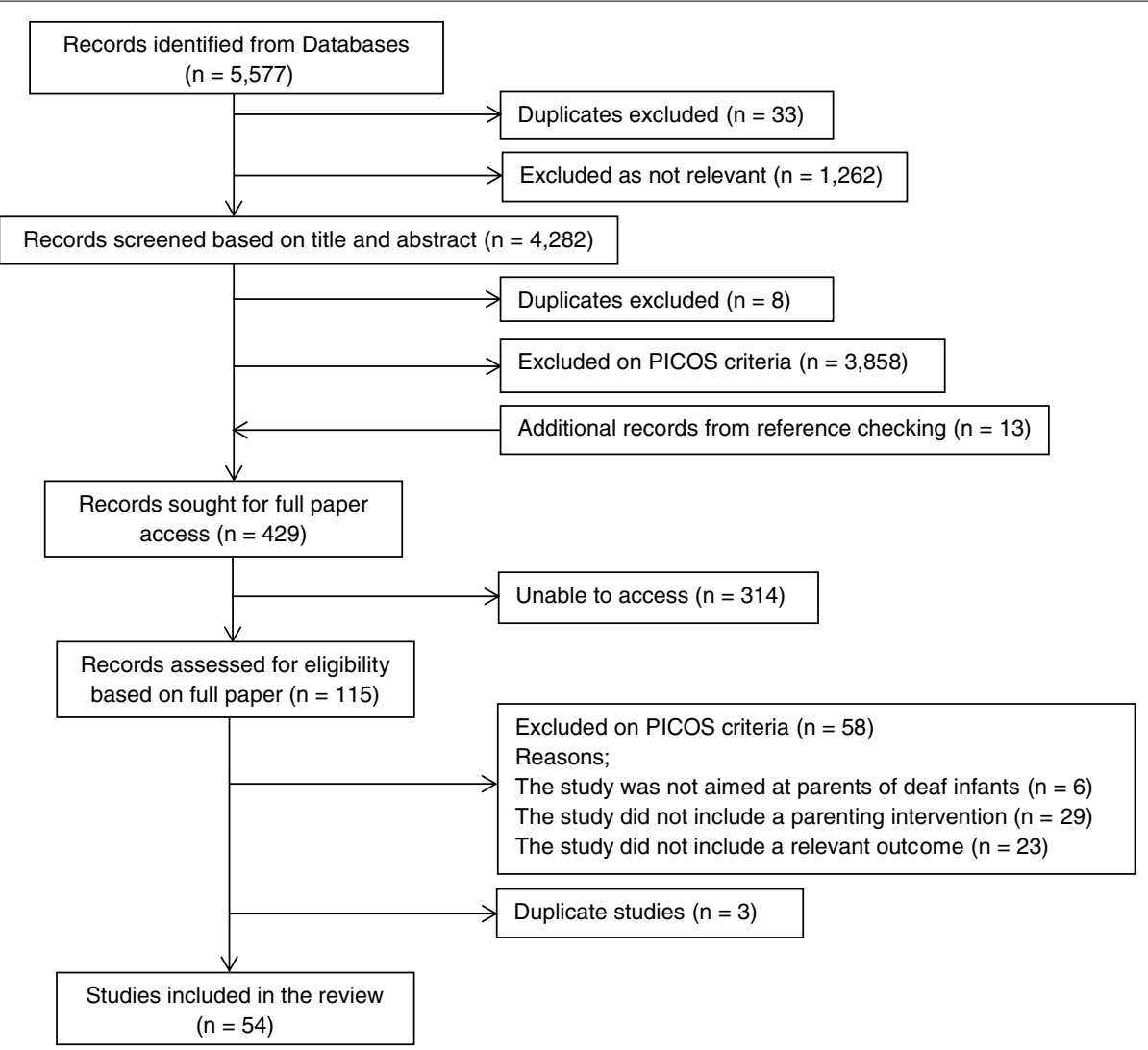

Fig. 1 PRISMA Diagram

\section{Results}

5577 records were identified through database searches. The review was completed by two independent reviewers over three sifts. The first two sifts examined records based on title and abstract. 41 were excluded by reviewers as duplications, 1,262 were identified as not relevant and 3,858 were excluded as they did not meet the PICOS criteria (see Fig. 1 for CONSORT). 13 additional records were added through reference checking. 429 papers were sought for full paper screening. If papers could not be accessed by the research team, we contacted relevant library services and the authors where necessary to obtain these. We had discussions with two authors to ensure we had captured relevant papers. There remained a number of papers which could not be accessed ( $\mathrm{n}=$ 314), potentially due to the lack of restriction on date. Reviewers conducted extensive reference checking to ensure no relevant papers were missed. 115 papers were included for full paper screening. 58 papers were excluded at this full paper third sift and a further 3 papers were excluded as duplicates. This left 54 included papers for data extraction.

Included study designs consisted of 5 randomised controlled trials, 17 non randomised comparison groups, 12 pre
/ post and 20 other designs (including longitudinal, qualitative evaluations, sub-analyses, file reviews, case studies).

Of those where the design enabled quality assessment (34 of the 54), 1 was deemed high-risk of bias, 4 had some concerns of bias, 18 were moderate risk, 10 low risk and one did not have sufficient detail to assess. For the remaining 20 studies, their designs did not allow for meaningful quality assessment to be conducted. Commonly identified issues included; lack of blinding, differences between groups at baseline (that was not controlled for), measures included self-report or observations and a lack of information provided on processes used for recruiting and allocating participants to groups.

Research outcomes were grouped by themes based on the primary focus of the intervention. Where there was an overlap between themes, the main outcome measures were considered to help categorise the studies. Four themes were identified; language and communication, parental wellbeing and empowerment, parental knowledge and skill and parent child relationship. These are discussed in further detail later.

The results are summarised in Table 1 (Table 1. Summary Results Table by Study Design) broken down by methodology, risk of bias and intervention focus. 
Table 1 Summary results table by study Design

\begin{tabular}{|c|c|c|c|}
\hline & Total Number & Risk of Bias & Theme \\
\hline RCTs & 5 & $\begin{array}{l}4 \text { some concerns } \\
1 \text { high risk }\end{array}$ & $\begin{array}{l}2 \text { language and communication } \\
3 \text { parent wellbeing and empowerment }\end{array}$ \\
\hline Comparison Group & 17 & $\begin{array}{l}14 \text { moderate } \\
2 \text { low } \\
1 \text { not enough information provided }\end{array}$ & $\begin{array}{l}9 \text { language and communication } \\
2 \text { parent wellbeing and empowerment } \\
4 \text { parent knowledge and skills } \\
2 \text { parent child relationship }\end{array}$ \\
\hline Pre-Post & 12 & $\begin{array}{l}4 \text { moderate } \\
8 \text { low }\end{array}$ & $\begin{array}{l}7 \text { language and communication } \\
3 \text { parent wellbeing and empowerment } \\
1 \text { parental knowledge and skills } \\
1 \text { parent child relationship }\end{array}$ \\
\hline Others & 20 & $N / A$ & $\begin{array}{l}11 \text { language and communication } \\
1 \text { parent wellbeing and empowerment } \\
8 \text { parental knowledge and skills }\end{array}$ \\
\hline Total & 54 & $\begin{array}{l}1 \text { high risk } \\
22 \text { moderate / some concerns } \\
10 \text { low } \\
1 \text { not enough information provided } \\
20 \text { N/A }\end{array}$ & $\begin{array}{l}29 \text { language and communication } \\
9 \text { parent wellbeing and empowerment } \\
13 \text { parental knowledge and skills } \\
3 \text { parent and child relationship }\end{array}$ \\
\hline
\end{tabular}

The most common target of intervention was language and communication (29 of the 54 studies). All 29 interventions in this category were found to have an effect, 17 of which were deemed significant although it is important to note methodological quality varied greatly.

13 interventions focused on parental knowledge and skills although there were several overlaps between this theme and the themes of parental empowerment and parent - child relationship. Intervention focus areas included skills for shared reading with the child (5 of the 13 interventions), techniques for managing child behaviour and hearing aid inspection.

9 interventions focused on parental wellbeing and empowerment. Parental wellbeing interventions largely looked at reducing parental stress and / or increasing parental support. Parental empowerment was often centred on decision making and accessing appropriate intervention as a way to reduce parental needs.

Only 3 interventions specifically focused on the parent child relationship. Two of these interventions found video feedback to be effective at increasing parental emotional availability although the results were not significant.

\section{Randomised Controlled Trials (RCTs)}

The following interventions were found to have a significant effect in randomised controlled trials:

\section{Parent-implemented communication treatment (PICT)}

This intervention includes strategies (visual, interactive, tactile and linguistic stimulation) to promote early communication (all forms including vocal, gestures and non-verbal) and parental sensitivity in everyday routines. In this study, parents in the treatment group increased their use of communication support strategies by $17 \%$ (effect size $1.08, \mathrm{p}=.04$ ). For children, larger gains were seen in pre-linguistic skills in treatment groups compared to control groups (effect size of 1.09, $\mathrm{p}=.03)[63]$.

\section{Muenster Parenting Programme}

This programme focuses on enhancing parents' responsive communicative behaviour to vocal and non-verbal signals of the child, and to reduce inappropriate parental behaviour that is too strongly initiative (e.g. inadequate introduction of a new action/object, neglecting the child's current focus of attention) [64]. There were significant increases in pre-post scores for trained parents in their responsiveness and a reduction in inappropriate initiative behaviour but not for the control group.

\section{Educational intervention program based on empowerment of mothers}

A training package was developed aiming to empower parents, by increasing their knowledge of interventions and improving their self-confidence [65]. This intervention focused on empowerment of parents, and also collected outcomes based on child speech development. Significant differences were seen in the intervention group's pre-post test scores for mothers' empowerment, self-efficacy, and adaptation. There was also a significant reduction for children's scores on the Newsha Developmental Speech Scale indicating a reduction in the severity of speech disorders. 
The final two RCTs tested 'Baby Portal' (a social network to facilitate discussion between parents) [66] and a 'Self-Instructional Parenting Program' (addressing feelings, attitudes and perceptions of interactions and teaching behaviour management principles to reduce stress) [67]. Neither found significant differences for those accessing the interventions.

\section{International Consensus Statement}

There is currently a 'Best Practices in Family-Centered Early Intervention for Children Who Are Deaf or Hard of Hearing: An International Consensus Statement' [48] of 10 principles which are deemed to be best practice for early intervention in this population. We mapped the interventions with RCT evidence to these 10 principles: however we found that none of the 5 RCTs included all 10 of the consensus principles. Commonly included was Principle 4: Family Social and Emotional Support, whereby families were provided with support systems to help them access knowledge and experience, and Principle 7: Qualified Providers, where those providing services to families had the necessary knowledge and experience. The included RCTs were less likely to report on Principle 3: Informed choice and decision making, relating to interventions where services provide families with the knowledge to make decisions based on special education laws and their rights and Principle 9: Progress Monitoring, relating to regular assessments of progress throughout the intervention. However, when comparing to the 10 consensus principles, we were only able to use available information about the intervention as provided in the papers, which were often limited in detail.

\section{Non-randomised or controlled group designs}

There were 9 studies focused on language and communication, 6 of which found a significant effect. These included studies which aimed to teach caregivers strategies to improve use of sign language and child vocabulary such as Adult "recasting" in sign language [68] and the ASPIRE Intervention Curriculum [69]. Auditory Verbal (AV) therapy [70] was also found to significantly improve language development in children using hearing technologies, and the Tri sensory language stimulation [71] found significant effects on nine out of nineteen variables related to language and vocabulary learning. Other studies included comprehensive training programmes, such as Bill Wilkerson Hearing and Speech Centre Program [72]. They found that children receiving early intervention had similar language competence to that of hearing children, and performed significantly better than those receiving late intervention. The Counselling and Home Training Program for Deaf Children [73] is a family oriented programme that encourages natural communication and building competence and esteem in a secure family context. The 1984 publication focuses on communication outcomes, with significantly more developmentally mature communication and high interaction in families who had received the intervention.

There were 3 studies which showed non-significant improvements from the intervention on the child's language and communication; these include the Tracy Clinic Oral Preschool [74]; a Parent Orientation Program [75] which focuses on early exposure to manual communication and The SKI*HI program [76] which teaches parents how to provide auditory and language stimulation for the child in the home.

4 studies focused on parental knowledge and skills, with significant results for a second study of PCIT [77] where reported outcomes focused on parent skills and managing child behaviour. There were also significant results for Interactive Storybook Reading [78], where the main outcomes focused on parent behavior, engagement, teacher techniques, and interactive reading. 2 studies showed non-significant improvements; the Deaf Mentorship Programme [79] where deaf adult mentors visit families to share their language, culture, and personal knowledge and the Parent Study Group [80] which is based on the Adlerian Model and helps parents manage their child's behaviour.

Both of the studies focused on parent wellbeing and empowerment showed significant results. These include The Counselling and Home Training Program for Deaf Children [81] which reports reduced parenting stress, and the family focused early intervention group [82] which focuses on home training for families in rural areas.

Both of the studies that focused on parent child relationships showed non-significant improvements in parental scores on the Emotional Availability Scales: Psychosocial video intervention, [83] and on the Gerrard parent-child questionnaire: Faranak parent-child program, [84].

\section{Pre/post study designs}

7 studies focused on language and communication, four of which showed significant results for increases in parent facilitation of spoken language: Parental Training Course, [85]; parent conversational ability Hanen based training program, [86]; child vocalisations ASPIRE intervention, [87]; and child hearing and speech skills Parent counselling, [88]. The other three studies showed nonsignificant improvements in learning and expression of language: Total communication, [89] and gains in child vocabulary SSE-2, [90]; Parent and child training, [91].

Of the 3 studies focused on parent wellbeing and empowerment, a group counselling programme showed 
significant improvements for parents: Parent Group Counselling Programme [92]. A remote intervention delivery service found no difference between tele intervention and conventional intervention in terms of communication performance of children meaning that telecommunication could be used as effectively as face to face; Tele intervention [93]. There were non-significant improvements for families in the HI HOPES [94] intervention, which has the central aim of informing and equipping parents to make their own decisions and measured parent satisfaction and child language outcomes.

The remaining 2 pre/post study designs focused on parental knowledge and skills, with a storybook reading programme reporting non-significant improvements: Parent child reading training [95] and on the parent child relationship, with a video feedback intervention showing non-significant maintenance and improvement on parent Emotional Availability scores: Video Interaction Guidance (VIG) [96].

\section{Other study designs}

For those studies that used other study designs, 11 focused on language and communication. Hogan et al. [97] predicted language scores based off modelling for Auditory-Verbal Therapy, but found no significant difference in rate of language development. Calderon and Low [98] conducted a sub-analysis of a larger study of Early Childhood Home Instruction and found non-significant improvements in language when a father was present at the intervention. Three studies looked at language communication using a longitudinal design; Oral Language Training [99]; Central Institute for the Deaf Early Education Project [100]; A Good Future for Deaf Children Programme [101], and all found improvements in language and communication for the child. There were also 6 papers that reported results from either case or file review, or case studies, all reporting improvements for the child's language; Auditory-Verbal Therapy [102]; The 10-year-old Early Childhood Home Instruction Program for Hearing-Impaired Infants and Their Families [103]; PiCS Intervention using distance education technology [104]; A program for teaching written language [105]; HI CHIPS Total Communication Program [106]; Communication Program [107].

8 studies focused on parental knowledge and skills including one logic model evaluation testing The Shared Reading Project [108]. There were 5 case studies, 3 showing positive changes for families; Parent Hearing Aid check training [109]; Educator-oriented contact intervention program [110]; Treatment Program [111]; one showing highly mixed results; Iowa E-Book [112], and one showing the intervention to be not effective at changing parent behaviour: Parent Training on Storybook Reading [113].

One study focused on parent wellbeing and empowerment using a retrospective survey design; Diagnostic Early Intervention Program (DEIP) [114], with findings showing family involvement and early enrolment as important factors for improving child outcomes.

The remaining 2 studies based on parental knowledge and skills were qualitative; Family-oriented early intervention program [115]; ASL Parent-Child Mother Goose [116], with both finding positive impacts for mothers.

\section{Discussion}

In summary, we found 5 RCTs which focussed mainly on parental wellbeing and empowerment $(n=3)$ or language and communication $(n=2)$. No RCTs were identified which tested interventions targeting the social and emotional development of the child. The categorisations of the themes of the studies are to be interpreted with caution, as there does remain overlap, with some papers fitting more than one theme. For example, some interventions describe the main aim of the programme to empower the parent in making decisions, but the main outcomes measured relate to child language development. The research team has aimed to group them based on the overarching intervention theme from the paper, to give the most meaningful overview of the current literature base in this field (as well as providing some type of comparison between a high number of included papers, which encompass a broad range of outcome measures and focuses). The large number of included studies was due to the PICOS criteria used being broad and very inclusive of a range of study parameters. This was intentional as the aim was to be able to provide a comprehensive overview of the current literature and be able to identify any gaps in research.

The largest gaps in the research appear to be threefold. Firstly there are only 5 randomised controlled trials of early parent interventions for deaf infants. Given that there are 34 million deaf children internationally, 5 RCTs is a remarkably small number of studies. Whilst there are some ethical challenges to carrying out RCTs in vulnerable populations this highlights the fact that we have little understanding of what kind of specialist support interventions can best improve outcomes for deaf infants.

Secondly the focus of the majority of the included interventions (29 of the 54) was on the language and communication development of the child or parental well-being, rather than wider aspects of the complex development and neurodevelopment of deaf children including for example their social and emotional development [117]. Language and communication is likely to be at the root of many of these difficulties particularly 
where infants have limited exposure to language learning $[26,27,118]$. The focus on language and communication is important but can lead parents to create a range of expectations, hopes and stressors related to this one area of development [119], and may have an impact on the way parents, families and professionals are enabled to have discussions about holistic aspects of quality of life. It may also inadvertently divert attention away from a range of other important aspects of development such as social and emotional development. The focus on language may also affect family stress and child outcomes. Indeed researchers reporting on language and literacy outcomes after the introduction of the UNHSP [120] have called for a broader range of outcomes to be attached to it [121] in order to better understand the broad range of developmental impacts on deaf children and their families. It would be a recommendation that current guidelines in this field, such as the International Guidelines for Best Practice [48] also emphasise a holistic approach, with a strong focus on social and emotional development of the child.

Thirdly, to scope the widest range of possible interventions the included literature spans from 1970 to 2019, with 12 published in the last 5 years. This mean that many of the programmes are less current and were conducted before the introduction of UNHSP, with very few examining issues of early intervention in terms of content or effect. The broad range of programmes also spans across cultural contexts [122] and so this may affect the way they are conceptualised, implemented, received and assessed, leading to a lack of generalisability. Many of the studies focused on short term outcomes, with few following outcomes in the medium to long term which would be useful to assess in any future research. There were also concerns over risk of bias in many of the studies assessed.

\section{Conclusion}

Training is necessary for support staff to be responsive to families' unique and often complex needs [123-125]. While UK QToDs receive guidance during training for working with early years children this is relatively limited, delivered within the full training protocol and centred around child development and language acquisition with the main focus of the course being centred around school aged children. Previously in the UK an additional qualification (Early Years ToDs PGDip/MA) in working with deaf babies under the age of two was available delivered by University of Hertfordshire/Mary Hare and many services have an Early Years (EYs) specialist QToD. The full course has now closed but it is still possible to complete individual modules. There is some very important work that needs to be done to understand what training is required, what parent support works well for different children in specific circumstances, how and when it should be delivered and to explore what evidence-based support interventions should be universally offered and what support is tailored to the individual needs of children and families. It is important to increase the availability of properly funded evidence based interventions and training opportunities to ensure that ToDs have a consistent approach to be able to provide individualised and responsive support to meet the widely varying needs of deaf children and their families.

The Joint Committee on Infant Hearing (JCIH) Position Statement 2007 expresses the goal of new-born hearing screening as 'to maximise linguistic competence and literacy development for children who are deaf or hard of hearing'. An international consensus statement has been released describing the principles for Family-Centered Early Intervention for Children Who Are Deaf or Hard of Hearing (of 10 principles) which are deemed to be of best practice for early intervention in this population $[48,126$, 127]. One potential gap in these statements is the relatively sparse mention of socio-emotional development and needs of deaf children.

The parent child relationship is important for the social and emotional development of deaf children [48] and further research could usefully explore how this may mediate outcomes and how it may be enhanced, including support for parents. Future research could also usefully include a wider set outcomes (alongside language development) such as theory of mind, empathy and executive functioning development, and collection of long term outcomes to reflect this. Since much of this research is before the advent of the UNHSP more current research is urgently needed.

\section{Abbreviations \\ CRIDE: Consortium for Research into Deaf Education; CRD: Centre for Reviews and Dissemination; dBHL: Decibels Hearing Loss; DEIP: Diagnostic Early Inter- vention Program; EYs: Early Years; JCIH: Joint Committee on Infant Hearing; NatSIP: National Sensory Impairment Partnership; PICT: Parent-implemented communication treatment; QToD: Qualified Teachers of the Deaf; RCTs: Ran- domised Controlled Trials; ROBINS-I: Risk Of Bias In Non-Randomised Studies of Interventions; TIDieR: Template for Intervention Description and Replica- tion; ToM: Theory of Mind; UNHSP: Universal Newborn Hearing Screening Programme; WHO: World Health Organisation.}

\section{Supplementary Information}

The online version contains supplementary material available at https://doi. org/10.1186/s12887-021-02893-9.

Additional file 1. Full search strategy.

Additional file 2: Table 1: Characteristics of Included Studies. 


\section{Acknowledgements}

The authors wish to acknowledge and thank the co-applicants of the NIHR funding application for their contribution to this project; Helen Phillips, Victoria Allgar, Dean McMillan, Danielle Varley, Alison Spear, Rachel Churchill.

We thank Kath Wright, an information specialist at the Centre for Reviews and Dissemination at the University of York, who helped develop the search strategy and ran the searches for the systematic review.

We also wish to thank the participants of the stakeholder groups and the parents who shared their valuable experiences of the diagnostic and early intervention process

\section{Authors' contributions}

Authors $\mathrm{RH}$ and $\mathrm{MG}$ conducted the systematic review, data extraction and appraisal. Authors BW, GC, TW, RS, IN and PS provided expert guidance in the subject area. All authors contributed and approved substantially to the writing of this paper.

\section{Funding}

This study/project is funded by the National Institute for Health Research (NIHR) [Research for Patient Benefit award PB-PG-1217-20041]. The views expressed are those of the author(s) and not necessarily those of the NIHR or the Department of Health and Social Care.

\section{Availability of data and materials}

All data generated or analysed during this study are included in this published article

\section{Declarations}

Ethics approval and consent to participate

Not applicable

\section{Consent for publication}

Not applicable

\section{Competing interests}

The authors declare that they have no competing interests.

\section{Author details}

${ }^{1}$ Leeds and York Partnership NHS Foundation Trust, COMIC Research, IT Centre, Science Park, University of York, Innovation Way, Heslington, York YO10 5NP, UK. ${ }^{2}$ The University College London Ear Institute, 332 Grays Inn Rd, London WC1X 8EE, UK. ${ }^{3}$ National Deaf Children's Society and NatSIP, Ground Floor South, Castle House 37-45 Paul Street, London EC2A 4LS, UK. ${ }^{4}$ University of Leeds, School of Education, Hillary Place, Woodhouse, Leeds LS2 9JT, UK. ${ }^{5}$ British Association of Teachers of the Deaf, 21, Keating Close, Rochester ME1 $1 \mathrm{EQ}, \mathrm{UK}$.

Received: 19 April 2021 Accepted: 3 September 2021

Published online: 22 October 2021

\section{References}

1. World Health Organisation, Deafness and Hearing Loss, 2021. https:// www.who.int/news-room/fact-sheets/detail/deafness-and-hearingloss. Accessed 1 Apr 2021.

2. CRIDE 2019. UK-wide summary report. https://www.ndcs.org.uk/media/ 6550/cride-2019-uk-wide-report-final.pdf. Accessed 21/05/2020

3. Mitchell RE, Karchmer M. Chasing the mythical ten percent: Parental hearing status of deaf and hard of hearing students in the United States. Sign Language Stud. 2004;4(2):138-63.

4. Moores DF. Educating the deaf: Psychology, principles, and practices. Boston: Houghton Mifflin Company; 2001.

5. National Deaf Childrens Society 2019. https://www.ndcs.org.uk/media/ 5542/ndcs_note_on_attainment_data_2019.doc. Accessed 21 May 2020

6. Roland L, Fischer C, Tran K, Rachakonda T, Kallogjeri D, Lieu JE. Quality of life in children with hearing impairment: systematic review and metaanalysis. Otolaryngol Head Neck Surg. 2016;155(2):208-19.
7. Action on Hearing Loss, 2015. About deafness and hearing loss statistics. https://www.england.nhs.uk/wp-content/uploads/2015/03/ act-plan-hearing-loss-upd.pdf. Accessed 24 March 2021

8. Eleweke CJ, Gilbert S, Bays D, Austin E. Information about support services for families of young children with hearing loss: A review of some useful outcomes and challenges. Deaf Educ Int. 2008;10(4):190-212.

9. Fitzpatrick E. Neurocognitive development in congenitally deaf children. Handb Clin Neurol. 2015;129:335-56.

10. Antia SD, Jones PB, Reed S, Kreimeyer KH. Academic status and progress of deaf and hard-of-hearing students in general education classrooms. J Deaf Stud Deaf Educ. 2009;14(3):293-311.

11. Barker DH, Quittner AL, Fink NE, Eisenberg LS, Tobey EA, Niparko JK, et al. Predicting behavior problems in deaf and hearing children: The influences of language, attention, and parent-child communication. Dev Psychopathol. 2009;21(2):373.

12. Dammeyer J. Psychosocial development in a Danish population of children with cochlear implants and deaf and hard-of-hearing children. J Deaf Stud Deaf Educ. 2010;15(1):50-8.

13. Niparko JK, Tobey EA, Thal DJ, Eisenberg LS, Wang NY, Quittner AL, et al. $\mathrm{CDaCl}$ Investigative Team. Spoken language development in children following cochlear implantation. Jama. 2010;303(15):1498-506.

14. Peterson CC, O'Reilly K, Wellman HM. Deaf and hearing children's development of theory of mind, peer popularity, and leadership during middle childhood. J Exp Child Psychol. 2016;149:146-58.

15. Schick B, De Villiers P, De Villiers J, Hoffmeister R. Language and theory of mind: A study of deaf children. Child Dev. 2007;78(2):376-96.

16. Turan Z. Supervision on Early Intervision Practices for Teachers of the Deaf. Educ Res Rev. 2019;14(11):388-96.

17. Hogan A, Shipley M, Strazdins L, Purcell A, Baker E. Communication and behavioural disorders among children with hearing loss increases risk of mental health disorders. Aust N Z J Public Health. 2011:35(4):377-83.

18. Nickbakht M. Supporting families of children with hearing loss transitioning to early intervention. Doctoral Thesis, University of Queensland; 2018

19. Tomblin JB, Oleson J, Ambrose SE, Walker EA, Moeller MP. Early literacy predictors and second-grade outcomes in children who are hard of hearing. Child Dev. 2020;91(1):e179-97.

20. Spencer PE, Marschark M. Evidence-based practice in educating deaf and hard-of-hearing students: Oxford University Press; 2010.

21. Hall ML, Eigsti IM, Bortfeld H, Lillo-Martin D. Executive function in dea children: Auditory access and language access. J Speech Language Hearing Res. 2018:61(8):1970-88.

22. Marschark M. Origins and interactions in the social, cognitive, and language development of deaf children. Psychol Perspect Deafness. 1993;1:7-26.

23. Garrido-Nag K, McCann J. Working With Deaf and Hard-of-Hearing Children From Linguistically Diverse Backgrounds. In: Cases on Communication Disorders in Culturally Diverse Populations; 2020. p. 157-79.

24. Knoors H, Marschark M. Teaching deaf learners: Psychological and developmental foundations: Oxford University Press; 2014.

25. Lederberg AR, Schick B, Spencer PE. Language and literacy development of deaf and hard-of-hearing children: Successes and challenges. Dev Psychol. 2013;49(1):15.

26. Hall ML, Eigsti IM, Bortfeld H, Lillo-Martin D. Auditory deprivation does not impair executive function, but language deprivation might: Evidence from a parent-report measure in deaf native signing children. J Deaf Stud Deaf Educ. 2017b;22(1):9-21.

27. Hall WC, Levin LL, Anderson ML. Language deprivation syndrome: A possible neurodevelopmental disorder with sociocultural origins. Soc Psychiatry Psychiatr Epidemiol. 2017a;52(6):761-76.

28. Dirks E. Psychosocial functioning in toddlers with moderate hearing loss: the importance of caregivers. Doctoral dissertation, Leiden University; 2018

29. Hintermair M, Sarimski K, Lang M. Preliminary evidence assessing social-emotional competences in deaf and hard of hearing infants and toddlers using a new parent questionnaire. J Deaf Stud Deaf Educ. 2017;22(2):143-54.

30. Margaret Brown P, Cornes A. Mental health of deaf and hard-ofhearing adolescents: What the students say. J Deaf Stud Deaf Educ. 2015:20(1):75-81. 
31. Vogel-Walcutt JJ, Schatschneider C, Bowers C. Social-emotional functioning of elementary-age deaf children: A profile analysis. Am Ann Deaf. 2011;156(1):6-22.

32. Scheetz NA. Psychosocial aspects of deafness: Allyn \& Bacon; 2004.

33. Rubin $\mathrm{KH}$, Bukowski WM, Parker JG. Peer interactions, relationships, and groups. Handbook Child Psychol. 2007;1:3.

34. Roberts S, Wright B, Moore K, Smith J, Allgar V, Tennant A, Doherty C, Hughes E, Moore DC, Ogden R, Phillips H. Translation into British sign language and validation of the strengths and difficulties questionnaire. Southampton: Review from NIHR Journals Library; 2015.

35. Bigler D, Burke K, Laureano N, Alfonso K, Jacobs J, Bush ML. Assessment and treatment of behavioral disorders in children with hearing loss: a systematic review. Otolaryngol Head Neck Surg. 2019;160(1):36-48.

36. Wood SA, Sutton GJ, Davis AC. Performance and characteristics of the Newborn Hearing Screening Programme in England: The first seven years. Int J Audiol. 2015;54(6):353-8.

37. Pimperton H, Kreppner J, Mahon M, Stevenson J, Terlektsi E, Worsfold S, et al. Language outcomes in deaf or hard of hearing teenagers who are spoken language users: Effects of universal newborn hearing screening and early confirmation. Ear Hear. 2017;38(5):598.

38. Sharma R, Gu Y, Ching TY, Marnane V, Parkinson B. Economic evaluations of childhood hearing loss screening programmes: a systematic review and critique. Appl Health Econ Health Policy. 2019;1:1-27.

39. Carr G. Screening: evaluating the outcomes of early intervention. ENT and Audiology News. https://www.entandaudiologynews.com/featu res/audiology-features/post/screening-evaluating-the-outcomes-ofearly-intervention. Accessed Feb 2021.

40. Yoshinaga-Itano C. From screening to early identification and intervention: Discovering predictors to successful outcomes for children with significant hearing loss. J Deaf Stud Deaf Educ. 2003;8(1):11-30.

41. Shekari E, Nakhshab M, Valinejad V, Modarres Zadeh A, Hosseinpour A. A Systematic Review of the Effectiveness of Early Intervention and the Role of Parents in Language Development of Hearing Loss Children. Iran Rehab J. 2017;15(1):5-14.

42. Joint Committee on Infant Hearing. Year 2007 position statement: Principles and guidelines for early hearing detection and intervention programs. Pediatrics. 2007;120(4):898-921.

43. Krishnan LA, Van Hyfte S, Richards KA. Newborn hearing screening: early education= more satisfied mothers. Am J Audiol. 2019;28(3):617-27.

44. Crowe K, Marschark M, Dammeyer J, Lehane C. Achievement, language, and technology use among college-bound deaf learners. J Deaf Stud Deaf Educ. 2017;22(4):393-401.

45. Jackson CW, Traub RJ, Turnbull AP. Parents' experiences with childhood deafness: Implications for family-centered services. Commun Disord Q. 2008;29(2):82-98.

46. Schmulian D, Lind C. Parental experiences of the diagnosis of permanent childhood hearing loss: a phenomenological study. Int J Audiol. 2020;59(1):54-60.

47. Cutler JM, Renda D, Lenzi G, Berrettini S. An effective collaboration between parent associations and medical professionals: The Italian Pediatric Federation Audiology Network. InItalian J Pediatrics. 2014;40(1):1 BioMed Central.

48. Moeller MP, Carr G, Seaver L, Stredler-Brown A, Holzinger D. Best practices in family-centered early intervention for children who are deaf or hard of hearing: An international consensus statement. J Deaf Stud Deaf Educ. 2013;18(4):429-45.

49. Yoshinaga-Itano C. Principles and guidelines for early intervention after confirmation that a child is deaf or hard of hearing. J Deaf Stud Deaf Educ. 2014;19(2):143-75.

50. Scarinci N, Gehrke M, Ching TY, Marnane V, Button L. Factors influencing caregiver decision making to change the communication method of their child with hearing loss. Deaf Educ Int. 2018;20(3-4):123-53.

51. Martin-Prudent A, Lartz M, Borders C, Meehan T. Early intervention practices for children with hearing loss: Impact of professional development. Commun Disord Q. 2016;38(1):13-23.

52. Public Health England 2018. https://www.gov.uk/government/publi cations/newborn-hearing-screening-programme-quality-standards. Accessed 21 May 2020

53. NatSIP Eligibility Framework Summer 2017 edition. https://www.natsip.org.uk/doc-library-login/eligibility-framework/00-eligibility-frame work-summer-2017-edition/916-eligibility-framework-document. Accessed 17 Feb 2021

54. NatSIP Eligibility Framework Summer 2019a edition. https://www natsip.org.uk/doc-library-login/eligibility-framework. Accessed 17 Feb 2021.

55. NatSIP Eligibility Framework, Early Years, 2019b [natsip.org.uk/doclibrary-login/eligibility-framework/eligibility-framework-for-deaf-andhi-children-early-years. Accessed 17/02/2021]

56. Grandpierre V. Barriers and Facilitators to Cultural Competence in Early Hearing Loss Services. Doctoral dissertation, University of Ottawa; 2018.

57. Espe-Sherwindt M, Serrano AM. It takes two: The role of familycentered practices in communication intervention. Revista de Logopedia, Foniatría y Audiología. 2016;36(4):162-9.

58. Wilson JM, Jungner G, World Health Organization. Principles and practice of screening for disease. Public Health Papers No. 34. 1968.

59. $\mathrm{JCIH}$, Joint Committee on Infant Hearing. Supplement to the $\mathrm{JCIH}$ 2007 position statement: Principles and guidelines for early intervention after confirmation that a child is deaf or hard of hearing. Pediatrics. 2013;131(4):e1324-e1349.

60. Hoffmann TC, Glasziou PP, Boutron I, Milne R, Perera R, Moher D, et al. Better reporting of interventions: template for intervention description and replication (TIDieR) checklist and guide. Bmj. 2014;7:348.

61. Higgins JP, Savović J, Page MJ, Elbers RG, Sterne JA. Assessing risk of bias in a randomized trial. Cochrane Handbook Syst Rev Interv. 2019;23:205-28.

62. Sterne JA, Hernán MA, Reeves BC, Savović J, Berkman ND, Viswanathan M, et al. ROBINS-I: a tool for assessing risk of bias in non-randomised studies of interventions. BMJ. 2016;12:355.

63. Roberts MY. Parent-implemented communication treatment for infants and toddlers with hearing loss: A randomized pilot trial. J Speech Language Hearing Res. 2019;62(1):143-52.

64. Glanemann R, Reichmuth $\mathrm{K}$, Matulat $\mathrm{P}$, am Zehnhoff-Dinnesen A. Muenster Parental Programme empowers parents in communicating with their infant with hearing loss. Int J Pediatr Otorhinolaryngol. 2013;77(12):2023-9.

65. Mostafavi F, Oryadi-Zanjani MM, Rad GS, Hazavehei SM, Rezaianzadeh A, Ravanyar L. Family-based training program: the role of mothers' empowerment in the speech development of children with hearing impairments. Pharmacophore. 2017;8(6S):e-1173273.

66. Aiello CP, Ferrari DV. Teleaudiology: efficacy assessment of an online social network as a support tool for parents of children candidates for cochlear implant. InCoDAS. 2015;27(5):411-8 Sociedade Brasileira de Fonoaudiologia.

67. Adams JW, Tidwell R. An instructional guide for reducing the stress of hearing parents of hearing-impaired children. Am Ann Deaf. 1989;1:323-8.

68. Prinz PM, Masin L. Lending a helping hand: Linguistic input and sign language acquisition in deaf children. Appl Psycholinguist. 1985;6(4):357-70

69. Suskind DL, Graf E, Leffel KR, Hernandez MW, Suskind E, Webber R, et al. Project ASPIRE: Spoken language intervention curriculum for parents of low-socioeconomic status and their deaf and hard-of-hearing children. Otol Neurotol. 2016;37(2):e110-7.

70. Hogan S, Stokes J, Weller I. Language outcomes for children of lowincome families enrolled in auditory verbal therapy. Deaf Educ Int. 2010;12(4):204-16.

71. Ivey LP, Teel JR. Tri-Sensory Language Stimulation With The TAVF Unit. Am Ann Deaf. 1974;1:318-20.

72. Mcconnell F. The parent teaching home: An early intervention program for hearing-impaired children. Peabody J Educ. 1974;51(3):162-70.

73. Greenberg MT, Calderon R, Kusche C. Early intervention using simultaneous communication with deaf infants: The effect on communication development. Child Dev. 1984;1:607-16.

74. Vernon M, Koh SD. Effects of oral preschool compared to early manual communication on education and communication in deaf children. Am Ann Deaf. 1971;1:569-74.

75. DeSalle Howse JM, Fitch JL. Effects of parent orientation in sign language on communication skills of preschool children. Am Ann Deaf. 1972;1:459-62. 
76. Watkins S. Long term effects of home intervention with hearingimpaired children. Am Ann Deaf. 1987;132(4):267-71.

77. Costa EA, Day L, Caverly C, Mellon N, Ouellette M, Wilson OS. Parentchild interaction therapy as a behavior and spoken language intervention for young children with hearing loss. Language Speech Hearing Serv Schools. 2019;50(1):34-52.

78. Dirks $E$, Wauters $L$. It takes two to read: interactive reading with young deaf and hard-of-hearing children. J Deaf Stud Deaf Educ. 2018:23(3):261-70.

79. Watkins S, Pittman P, Walden B. The deaf mentor experimental project for young children who are deaf and their families. Am Ann Deaf. 1998;143(1):29-34.

80. Perry D. Parenting a hearing impaired child: an Adlerian approach Doctoral dissertation, The University of Arizona; 1986.

81. Greenberg MT. Family stress and child competence: The effects of early intervention for families with deaf infants. Am Ann Deaf. 1983:1:407-17.

82. Kargin T. Effectiveness of a family-focused early intervention program in the education of children with hearing impairments living in rural areas. Int J Disabil Dev Educ. 2004;51 (4):401-18.

83. Lam-Cassettari C, Wadnerkar-Kamble MB, James DM. Enhancing parent-child communication and parental self-esteem with a video-feedback intervention: outcomes with prelingual deaf and hard-of-hearing children. J Deaf Stud Deaf Educ. 2015;20(3):266-74.

84. Koohi R, Sajedi F, Movallali G, Dann M, Soltani P. Faranak Parent-Child Mother Goose Program: Impact on Mother-Child Relationship for Mothers of Preschool Hearing Impaired Children. Iran Rehabil J. 2016;14(4):201-10.

85. Harrigan S, Nikolopoulos TP. Parent interaction course in order to enhance communication skills between parents and children following pediatric cochlear implantation. Int J Pediatr Otorhinolaryngol. 2002;66(2):161-6

86. Paganga S, Tucker E, Harrigan S, Lutman M. Evaluating training courses for parents of children with cochlear implants. Int J Language Commun Disord. 2001;36(S1):517-22.

87. Sacks C, Shay S, Repplinger L, Leffel KR, Sapolich SG, Suskind E, et al. Pilot testing of a parent-directed intervention (Project ASPIRE) for underserved children who are deaf or hard of hearing. Child Language Teach Ther. 2014;30(1):91-102.

88. Ling AH. Changes in the abilities of deaf infants with training. J Commun Disord. 1971;3(4):267-79.

89. Dee A, Rapin I, Ruben RJ. Speech and language development in a parent-infant total communication program. The Annals of otology, rhinology \& laryngology. Supplement. 1982;97:62-72.

90. Luetke-StahIman B, Moeller MP. Enhancing parents' use of SEE-2: Progress and retention. Am Ann Deaf. 1990;135(5):371-8.

91. Lund $E$. The effects of parent training on vocabulary scores of young children with hearing loss. Am J Speech Language Pathol. 2018;27(2):765-77.

92. Backenroth G. Counselling in families with a deaf or hearing impaired child. Int J Adv Couns. 1984;7(4):267-74.

93. Havenga E, Swanepoel DW, Le Roux T, Schmid B. Tele-intervention for children with hearing loss: A comparative pilot study. J Telemed Telecare. 2017:23(1):116-25.

94. Störbeck C, Pittman P. Early intervention in South Africa: Moving beyond hearing screening. Int J Audiol. 2008;47(sup 1):S36-43.

95. Nelson LH, Stoddard SM, Fryer SL, Muñoz K. Increasing engagement of children who are $\mathrm{DHH}$ during parent-child storybook reading. Commun Disord Q. 2019;41(1):12-21.

96. James DM, Wadnerkar-Kamble MB, Lam-Cassettari C. Video feedback intervention: a case series in the context of childhood hearing impairment. Int J Language Commun Disord. 2013;48(6):666-78.

97. Hogan S, Stokes J, White C, Tyszkiewicz E, Woolgar A. An evaluation of auditory verbal therapy using the rate of early language development as an outcome measure. Deaf Educ Int. 2008;10(3):143-67.

98. Calderon R, Low S. Early social-emotional, language, and academic development in children with hearing loss: Families with and without fathers. Am Ann Deaf. 1998;1:225-34.

99. Suzuki S, Notoya M. Teaching written language to deaf infants and preschoolers. Top Early Child Spec Educ. 1984;3(4):10-6.

100. Simmons-Martin A. Efficacy report: Early education project. J Div Early Childhood. 1981;4(1):5-10.
101. Takala M, Kuusela J, Takala EP. " A good future for deaf children": A fiveyear sign language intervention project. Am Ann Deaf. 2000;1:366-74.

102. Easterbrooks SR, O'Rourke CM, Todd NW. Child and Family Factors Associated With Deaf Children's Success in Auditory-Verbal Therapy. Otol Neurotol. 2000;21(3):341-4.

103. Thompson MD, Swisher MV. Acquiring language through total communication. Ear Hear. 1985;6(1):29-32.

104. Daczewitz ME. Delivering the parent-implemented communication strategies (PICS) intervention using distance training and coaching with a father and his child who is hard of hearing. Illinois State University; 2015.

105. Steinberg DD. Overcoming linguistic limitations of hearing-impaired children through teaching written language. Topics Lang Disord. 1982;2(3):17-28. https://doi.org/10.1097/00011363-198206000-00007.

106. Gaines R, Halpern-Felsher BL. Language preference and communication development of a hearing and deaf twin pair. Am Ann Deaf. 1995; 1:47-55

107. Seitz S, Marcus S. Mother-child interactions: A foundation for language development. Except Child. 1976;42(8):445-9.

108. Delk L, Weidekamp L. Shared Reading Project: Evaluating Implementation Processes and Family Outcomes. Sharing Results. National Deaf Education Network. Gallaudet Univ; 2001.

109. Foust TE, Wynne MK. Effectiveness of supplemental parent training in hearing aid checks. J Acad Rehab Audiol. 1991:24:85-96.

110. Janssen MJ, Riksen-Walraven JM, Van Dijk JP. Contact: Effects of an intervention program to foster harmonious interactions between deaf-blind children and their educators. J Visual Impairment Blindness. 2003;97(4):215-29.

111. Garrard KR, Saxon SA. Preparation of a disturbed deaf child for therapy: A case description in behavior shaping. J Speech Hear Disord. 1973:38(4):502-9.

112. Mueller VT. The effects of a fluent signing narrator in the lowa E-Book on deaf children's acquisition of vocabulary, book related concepts, and enhancement of parent-child lap-reading interactions. The University of lowa; 2008.

113. Bergeron JP. Effectiveness of parent training on shared reading practices in families with children who are deaf and hard of hearing. Georgia State University; 2013.

114. Moeller MP. Early intervention and language development in children who are deaf and hard of hearing. Pediatrics. 2000;106(3):e43.

115. Dromi E, Ingber S. Israeli mothers' expectations from early intervention with their preschool deaf children. J Deaf Stud Deaf Educ. 1999;4(1):50-68.

116. Snoddon K. American Sign Language and early literacy: Research as praxis: University of Toronto; 2009

117. Vissers CT, Hermans D. Social-emotional problems in deaf and hardof-hearing children from an executive and theory of mind perspective. Evid Based Pract Deaf Educ. 2018;28:455-76.

118. Meristo M, Hjelmquist E, Morgan G. How access to language affects theory of mind in deaf children. In: Access to language and cognitive development; 2012. p. 44-61.

119. Young A, Tattersall H. Universal newborn hearing screening and early identification of deafness: Parents' responses to knowing early and their expectations of child communication development. J Deaf Stud Deaf Educ. 2007:12(2):209-20.

120. Pimperton H, Blythe H, Kreppner J, Mahon M, Peacock JL, Stevenson $J$, et al. The impact of universal newborn hearing screening on longterm literacy outcomes: a prospective cohort study. Arch Dis Child. 2016;101(1):9-15.

121. Pimperton $\mathrm{H}$, Kennedy $\mathrm{CR}$. The impact of early identification of permanent childhood hearing impairment on speech and language outcomes. Arch Dis Child. 2012;97(7):648-53.

122. Erting CJ. Cultural conflict in a school for deaf children. Anthropol Educ Q. 1985:16(3):225-43.

123. Melanson S. Family Centred Practices in Early Intervention in Nova Scotia: Quality of Life Issues for Families, 140.230.24.101. Doctoral dissertation, 2007.

124. Poon BT, Zaidman-Zait A. Social support for parents of deaf children: Moving toward contextualized understanding. J Deaf Stud Deaf Educ. 2014;19(2):176-88. 
125. Wood Jackson C, Turnbull A. Impact of deafness on family life: A review of the literature. Top Early Child Spec Educ. 2004;24(1):15-29.

126. Danermark B, Granberg S, Kramer SE, Selb M, Moller C. The creation of a comprehensive and a brief core set for hearing loss using the international classification of functioning, disability and health. Am J Audiol. 2013. https://doi.org/10.1044/1059-0889(2013/12-0052).

127. Muse C, Harrison J, Yoshinaga-Itano C, Grimes A, Brookhouser PE, Epstein S, et al. Supplement to the JCIH 2007 position statement:
Principles and guidelines for early intervention after confirmation that a child is deaf or hard of hearing. Pediatrics. 2013;131(4):e1324-49.

\section{Publisher's Note}

Springer Nature remains neutral with regard to jurisdictional claims in published maps and institutional affiliations.
Ready to submit your research? Choose BMC and benefit from:

- fast, convenient online submission

- thorough peer review by experienced researchers in your field

- rapid publication on acceptance

- support for research data, including large and complex data types

- gold Open Access which fosters wider collaboration and increased citations

- maximum visibility for your research: over $100 \mathrm{M}$ website views per year

At BMC, research is always in progress.

Learn more biomedcentral.com/submissions 